\title{
Young Flames: The Effects of Childhood Exposure to Fire on Adult Attitudes
}

\author{
Damian R. Murray \\ and Daniel M. T. Fessler \\ University of California, Los Angeles
}

\author{
Gwen Lupfer \\ University of Alaska Anchorage
}

\begin{abstract}
Successful use of fire has been essential to survival throughout the majority of human history - an environmental pressure that may have led to cognitive mechanisms dedicated to attaining mastery of fire manipulation and control. Concordant with this hypothesis is the fact that, despite its inherent danger, the frivolous use of fire remains firmly embedded within modern societies; conversely, in societies where fire is used for utilitarian purposes, ethnographic reports suggest that fire is considered mundane. The exposure hypothesis holds that the attraction to fire in modern societies is due to the lack of adequate exposure to fire throughout childhood. Two studies-comprising North American samples that have had significantly different levels of exposure to fire-investigated the relationship between frequency of exposure to fire throughout childhood and psychological associations with fire. Psychological associations with fire were overwhelmingly positive in both samples. Study 1 found no significant association between childhood fire exposure and positive affective associations with fire. Using a more sophisticated measurement tool and in a more rural sample, Study 2 found that, contrary to the exposure hypothesis, more frequent exposure to fire in childhood was associated with more positive psychological associations with fire. Potential reasons for the discrepancies between these results and earlier ethnographic reports, and their potential implications, are discussed.
\end{abstract}

Keywords: cultural transmission, evolution, fire, prepared learning, social learning

The control and manipulation of fire has played a central role in human evolution. Although debate exists over the earliest evidence of hominids' controlled use of fire, reliable physical evidence from multiple regions has been dated at 700,000 years ago and earlier (Berna et al., 2012; Goren-Inbar et al., 2004),

This article was published Online First January 12, 2015.

Damian R. Murray, Department of Psychology, University of California, Los Angeles; Daniel M. T. Fessler, Department of Anthropology, University of California, Los Angeles; Gwen Lupfer, Department of Psychology, University of Alaska Anchorage.

This research was supported by a postdoctoral fellowship (award 756-2013-0521) awarded to Damian R. Murray by the Social Sciences and Humanities Research Council of Canada.

Correspondence concerning this article should be addressed to Damian R. Murray, Department of Psychology, University of California, Los Angeles, 1285 Franz Hall, Los Angeles, CA 90095-1563. E-mail: dmurray@psych.ucla .edu with many more speculative pieces of evidence surpassing the 1 million year mark (Brain, 1993; Sillen \& Hoering, 1993). Regardless of the exact date of controlled fire use, fire clearly served as a necessary tool throughout the evolutionary history of Homo sapiens.

The control of fire provided several strategic advantages for humans. Fire provides warmth, light, a weapon of use against predators or rival groups, and a tool to manipulate objects and the natural environment (Burton, 2009; Pyne \& Goldammer, 1997). Controlled fire use provided critical nutritional breakthroughs as well, as cooking allowed for calories to be gleaned from otherwise indigestible foods, and also substantially reduced exposure to foodborne pathogens. Cooking, in turn, had implications for human physiology: the caloric efficiency of cooked food reduced the energetic costs of the gut and created selection for shorter guts and larger brains (Wrangham, 2009). Dependence upon fire may have also had social implications. 
Burton (2009) suggested that the additional light provided by fire in the evening offers extended opportunities for communication, group coordination, and transmission of cultural norms. For example, whereas daytime conversations among the Ju/'hoan (!Kung) Bushmen of South Africa focus predominantly upon economic and pragmatic political matters, evening fireside conversations predominantly involve stories that convey information about cultural institutions and social networks (Wiessner, 2014). This multifaceted utility of fire facilitated the radiation of anatomically (and psychologically) modern humans across the globe.

Of course, the use of fire is not without its costs. Along with the acute causes of death and injury from mishandled fires, chronic exposure to even low levels of smoke from wood and other biomass has several adverse health consequences (for review, see Naeher et al., 2007). Indeed, research suggests that the ability to tolerate smoke from wood combustion was selected for in populations that relied heavily upon fire for heat (Platek, Gallup, \& Fryer, 2002). However, despite the fact that the use and control of fire remains universal (Brown, 1991), and despite its necessity and probable force as a selective pressure across much of human history, empirical investigations into the psychology underlying fire use are surprisingly scant.

\section{Contemporary Interactions With Fire}

Although open fires provide no practical benefit within contemporary industrialized societies, their use for aesthetic and ceremonial purposes remains firmly embedded in these societies' cultural and psychological fabric. Despite strict regulatory controls on all things fire-related, fire use still comes with immense cost. In the United States, for example, fires in residences from candles alone cause about $\$ 390$ million in property loss annually; between December 24 and 26 alone there are an estimated 11,600 Christmas tree-related fires annually that require fire department response. Fire deaths from all causes in the United States numbered almost 40,000 between 2001 and 2010 (www.usfa.fema .gov), making it the third leading cause of accidental home injury deaths. The danger that fire represents appears to do nothing to curb the appetite for fire within residences: There are an es- timated 1.2 fireplaces per American home, and the country's fireplace sales and instillations represented a $\$ 5$ billion industry in 2011 (www. hpba.org), although the use of these fireplaces may admittedly be infrequent.

Children and adolescents appear to be particularly interested in fire. Issues of public safety have spurred the majority of studies examining children's interest in fire, with the focus correspondingly placed primarily upon deviant fire setting and its potential pathological underpinnings. These investigations are certainly justified, as child and adolescent fire play results in thousands of injuries and deaths annually in developed countries (see, e.g., Hall, 2003). Several surveys offer a portrait of the prevalence of childhood interest in - and play with-fire. One study of 95 Swedish 18-year olds, for example, found that $70 \%$ of men and $44 \%$ of women reported having played with fire during childhood (Perrin-Wallqvist \& Norlander, 2003). Similarly, a survey of over 5,000 Oregonian elementary schoolchildren found that $47 \%$ reported having played with fire-and $10 \%$ reported having started their first fire before age five (Simonsen \& Bullis, 2001). A similar survey of Canadian students found that, by Grade $13,81 \%$ of students reported having played with fire (Cotterall, 2003). Pinsonneault (2002) examined the records of fires involving children in Oregon over a 4-year period. Records from 4,600 fires revealed that 12-year-olds were responsible for the highest proportion of fires. These data further showed an increase in involvement from ages 1 to 12 and, interestingly, a consistent decrease in involvement in the years after 12 .

Some of these studies have also assessed affective associations with fire. PerrinWallqvist, Archer, and Norlander (2004), for example, found in a sample of Swedish 16year-olds that $61 \%$ of participants thought fire was "cozy," and $20 \%$ found fire "exciting." Only $9 \%$ of this sample reported that they found fire "uninteresting." However, aside from attempts to link generalized conduct disorders to deviant fire play (e.g., Kolko, 2002; Ó Ciardha \& Gannon, 2012), very little work has investigated the developmental precursors of interest in fire. 


\section{Cognitive Biases Toward Learning About Fire?}

One notable exception to this paucity of firebased research is Fessler (2006), who suggested that, because of the recurrent selective pressure of fire over hundreds of thousands of years of human history, humans may have dedicated cognitive mechanisms for learning about fire. These mechanisms, in turn, may be responsible for the intrinsic interest in fire seen in individuals within industrialized societies. Consistent with this idea are empirical results suggesting the presence of equivalent mechanisms dedicated toward learning about other fitnessrelevant properties of ancestral environments, such as potentially toxic foods (Garcia, Ervin, \& Koelling, 1966), predatory and venomous animals (Barrett \& Broesch, 2012; Broesch, Barrett, \& Henrich, 2014; Ohman, Fredrikson, Hugdahl, \& Rimmo, 1976), and methods of disease transmission and contamination (see Siegal, Fadda, \& Overton, 2011). Fessler suggested that an information acquisition mechanism dedicated to fire learning should be similar to that of a predator-information acquisition system for at least two reasons. First, like predators, the specific shape, size, and other physical properties of combustible fuels are not universal. Second, pure trial-and-error learning in both of these domains is likely not appropriate, given that the fitness consequences of negative feedback (in the form of a serious bite or burn, respectively) may be too great. Therefore, the specific properties of a fire-learning machinery should be similar to those of a predatorinformation acquisition system, such as learning occurring without extrinsic motivation, rapid information acquisition, heavy reliance on socially transmitted information, and skill acquisition through intrinsically motivated emulation (from Barrett, 2005).

Inspired by his own observations in rural Southwestern Sumatra, where fire is simply used as a tool, and where none of the Westerntype fascination toward fire exists, Fessler (2006) obtained retrospective reports from 18 ethnographers, working in 19 small-scale societies, regarding typical fire-related behaviors and attitudes toward fire. These retrospective observations offered several interesting pieces of evidence. First, children typically begin interacting with fire before age 6 , and gain profi- ciency with starting and maintaining a fire just before age 7. Second, with limited exception, children do not appear to use fire for entertainment purposes. For adults in these societies, fire appears to be viewed only in mundane, utilitarian terms; no ethnographers reported fire gazing to be typical in either children or adults. Based on these reports, Fessler (2006) suggested that the preoccupation with fire in contemporary societies may be due to the relative novelty of largely fire-free environments in childhoodadult fascination with fire may be an artifact of the stunted cognitive machinery dedicated to learning about the properties of fire.

\section{A Relationship Between Fire Exposure and Interest?}

The limited research reviewed above suggests two tentative conclusions. First, Western children, adolescents, and adults-populations that receive little exposure to fire in their dayto-day lives relative to people in preindustrialized societies-appear to be intrinsically interested in fire. Throughout these life stages, this interest manifests in questions about the categorical properties of fire-relevant materials, potentially dangerous fire play, and dedicating vast economic resources to increasing access to fire, respectively. A second tentative conclusion comes from ethnographic reports of traditional preindustrial societies-where fire use is common and utilitarian-which suggest that in these societies fire interest peaks in early childhood but declines rapidly thereafter, presumably once mastery of fire skills is attained. These two bodies of evidence imply a causal relationship between the level of fire exposure and interest in fire: Greater levels of exposure in early childhood should negatively predict levels of interest in adulthood. To date, however, systematic investigations of this relationshipwhat we term the exposure hypothesis-are absent in the literature.

\section{Overview of the Present Investigation}

This article presents the results of the first systematic investigations into the relationship between childhood fire exposure and adult interest in fire. Study 1 reports preliminary evidence relevant to this question from an other- 
wise unrelated study carried out in Vancouver, Canada. Study 2 was designed specifically for the purposes of investigating this question. In planning this second study, we were mindful that the characteristics of the study population are paramount in addressing this question-to avoid floor and ceiling effects, the population should not be characterized by uniform constant exposure to fire, nor should it be characterized by uniform lack thereof; to maximize the probability of detecting a relationship, sufficient variation should exist in both the hypothesized independent and dependent variables. Therefore, our second sample was drawn from a population in Anchorage, Alaska. We obtained detailed information regarding participants' childhood interactions with fire, along with their current interest in, and affective associations with, fire.

\section{Study 1}

\section{Method}

Participants. Participants were 125 students (18 men) enrolled at the University of British Columbia, Vancouver, Canada (average age $=20.5$ years, $S D=3.6$ ). The sample was ethnically diverse: $50.4 \%$ reported being of East Asian descent, $33.6 \%$ of European descent, $5.6 \%$ of South Asian descent, and $10.4 \%$ of other ethnicities. All participants completed the questionnaire alone in a laboratory room.

Materials. Participants completed a set of questionnaires assessing demographic information and several other measures unrelated to the current investigation. Relevant to the current investigation, five questions assessed fire exposure and behavioral and psychological experiences with small fires (defined as campfires, cooking fires, and hearth fires). The first two questions assessed average frequency of exposure to these types of fires before age 10, and between ages 10 and 18. Response options were a few times a week or more, a few times a month, a few times a year, a few times during my entire childhood, and never. A third question assessed the number of times participants had been in the presence of a small fire in the past year, in free-response format. A fourth question asked participants, in free-response format, to "please list any feelings or emotions you typically associate with small fires." A final ques- tion asked participants if they have had any negative experiences with fire (by circling yes/ no), and, if so, to describe this/these negative experiences.

\section{Results and Discussion}

Consonant with the predominantly urban background of students at this university, preliminary analyses revealed that participants in this sample had relatively little exposure to small fires before 10 years of age: $9.6 \%$ reported being in the presence of fire at least a few times a week, $11.2 \%$ a few times a month, $37.6 \%$ a few times a year, $32.8 \%$ a few times during the entire time period, and $8.8 \%$ never having been in the presence of a small fire. Within this sample, men reported somewhat greater exposure than women (e.g., whereas $33.3 \%$ of men reported being in the presence of fire a few times a month or more, $18.6 \%$ of women reported this frequency). However, statistical comparisons between men and women are constrained by the very low proportion of men, and this difference was not statistically significant $(p=.16)$, nor was the overall reported frequency pattern significantly different, $p>.50$.

Affective associations with fire. Our first analyses investigated participants' affective associations with fire. Participants listed an average of $2.60(S D=1.12)$ emotions/feelings in response to Question 4, ranging from 0 to 5 responses. We sorted each of these responses into positively valenced (e.g., happy, socially connected, content), negatively valenced (e.g., fear, caution), and neutral (e.g., "nothing," hunger, spirituality). Although "warmth" was frequently listed, because this term's meaning is ambiguous, it was not included as a positive affective response for initial analyses. In total, $69.4 \%$ of participants listed at least one positively valenced affective reaction (if "warmth" was included in this category, this percentage rose to $91.7 \%$ ). A nonsignificantly higher proportion of women $(70.8 \%)$ than men $(61.1 \%)$ listed a positively valenced affective reaction $(p=.41)$. Conversely, $16.5 \%$ of participants listed at least one negatively valenced affective response, a proportion that was nonsignificantly higher for men $(22.2 \%)$ than women $(15.5 \%$; $p=.49$ ). Just $5.9 \%$ of participants reported neutral affective reactions. The difference be- 
tween the proportion of participants listing positively versus negatively valenced affective reactions was highly significant, $t=8.22, p<$ .001 (and significant when considering men and women separately, $p s<.05$ ).

Does childhood exposure to fire predict affective reactions in adulthood? The central analyses of interest investigated whether frequency of exposure to fire in childhood was related to the likelihood of reporting positive associations with fire. Frequency of exposure was used as a continuous variable in a singlepredictor binary logistic regression predicting whether a participant listed at least one positive affective association with fire. Exposure did not emerge as a significant predictor of positive association, $B=.25, p=.19$. Inconsistent with the exposure hypothesis, the direction of this relationship was toward more exposure being associated with more positive valencealthough this relationship was nonsignificant for both men $(p=.16)$ and women $(p=.43)$. Frequency of childhood exposure was unrelated to the probability of negative associations with fire $(B=0.02, p=.95)$.

Although the results of Study 1 are inconsistent with the exposure hypothesis, the nature of the sample used is such as to limit the strength of this test, as most participants had very little exposure to fire early in life. To address this limitation, we conducted a second, more exhaustive study, sampling a student population in Anchorage, Alaska, a region with greater quotidian reliance on fire.

\section{Study 2}

\section{Method}

Participants. Participants were 231 students (40 men) enrolled at the University of Alaska, Anchorage (mean age 24.4 years, $S D=$ 7.4). The reported ethnic background of the sample was $64.5 \%$ European, 9.2\% Native American, 7.5\% Black, 5.3\% Asian, 3.9\% Latino, $6.6 \%$ mixed ethnicity, and $3.1 \%$ other ethnicities. Of this sample, $40.4 \%$ reported being born in Alaska, 51.8\% in another American state, and $7.9 \%$ elsewhere. All participants completed the questionnaires online in exchange for partial course credit.

Materials. Participants completed a set of measures assessing interactions with, and psychological reactions to, small wood fires (defined as campfires, cooking fires, and hearth fires, but not artificial fires such as gas-fueled fires, stoves, lighters, or cigarettes). Similar to Study 1, the central measure assessing exposure to fire asked participants to report how often they were typically in the presence of this type of fires before age 10 (response options were almost daily, a few times a week, a few times a month, a few times a year, a few times during my entire childhood, and never). Further, participants listed, in free-response form, specifically what types of fires they were exposed to during this time. Participants also reported their interest in fire in childhood on an ordinal scale (ranging from extremely interested to not at all interested), whether they used fire for entertainment purposes during this period, and whether they were ever injured from using fire this way. Each of these questions were also asked for the period between the ages of 10 and 18 years, and also for the period of the past year. Participants also reported their perceived proficiency with lighting a wood fire, describing how easily the feel they could light a wood fire without the aid of artificial combustibles (response options ranged from very easily to I could not light a fire in this way). As in Study 1, participants were also asked, in free-response format, to "please list any feelings or emotions you typically associate with small fires" (because of a computer error only 121 of the participants received this question). The final question asked participants if they have had any negative experiences with fire (by circling yes/no), and, if so, to describe this/these negative experiences.

Participants also completed a 10-item scale designed to assess general positive psychological associations with, and interest in, fire. Example items include "sitting close to a small fire makes me happy;" "watching a fire does not interest me" (reverse coded). Agreement with each of these items was assessed on a 7-point Likert scale ranging from 1 (strongly disagree) to 7 (strongly agree). Preliminary analyses demonstrated that this scale was internally reliable (Cronbach's alpha = .91 ), and that although men reported slightly higher positivity scores than women, this difference was nonsignificant (5.01 vs. $4.75, p=$ $.27)$. 


\section{Results}

Participants in the Anchorage sample reported relatively greater exposure to fire before the age of 10 than did participants in Study 1. In the current sample, $8.5 \%$ reported being in the presence of small fires almost daily, $13.2 \%$ a few times a week, $23.9 \%$ a few times a month, $38 \%$ a few times a year, $11.1 \%$ a few times during this entire period, and 5.1\% "never." Overall, whereas only $20.8 \%$ of the participants in Study 1 reported being in the presence of fire at least a few times a month during childhood, $45.7 \%$ of the current sample reported this frequency. This frequency did not significantly differ between men and women $(52.6 \%$ vs. $44.5 \%$ respectively, $p=.36$ ), nor did the overall reported frequency pattern significantly differ $(p>.50)$. Also, a higher proportion of men than women reported that it would be "somewhat easy" or "very easy" to light a wood fire without artificial combustibles (68.4\% vs. $52.9 \%)$, but this difference was only marginally significant, $p=.079$.

When specifying (free-response) what types of fires these typically were, $76 \%$ of participants reported that the fires were typically open fires (campfires, bonfires), and 36\% reported that the fires were closed fires (hearth fires, stoves; many participants listed more than one type of fire). Thirty-two percent of participants specifically alluded to being exposed to fires used for cooking.

We were attentive to the possibility that participants actually born in Alaska might have different experiences with fire than participants who were not. However, preliminary analyses revealed no meaningful differences between these groups: native-born Alaskans did not differ from those who immigrated to Alaska on frequency of childhood exposure to fire, fire exposure in the past year, reported proficiency using fire, childhood interest in fire, current interest in fire, or probability of listing a positively or negatively valenced affective associations (all $p s>.20$ ). Thus, these participants were not treated separately in the analyses reported below.

Affective reactions to fire. Participants listed an average of $2.24(S D=1.62)$ emotions/ feelings when asked (free response) about their affective associations with fire, ranging from 0 to 8 responses. Similar to Study 1, we sorted these responses into positively valenced (happiness, safety, interpersonal closeness, contentment) and negatively valenced (sadness, fear, anxitety). Again, neither "warmth" nor less clearly valenced responses (e.g., hunger, fascination) were placed into these categories. Similar to Study 1, $74.1 \%$ of participants listed at least one positively valenced affective association $(77.3 \%$ of men vs. $73.0 \%$ of women, $p>$ $.50)$. On the other hand $8 \%$ listed at least one negative affective association ( $4.5 \%$ of men vs. $8.9 \%$ of women, $p>.50$ ), and $8.8 \%$ listed neutral or no affective associations (e.g., "nothing," "hunger").

Does childhood exposure to fire predict affective reactions in adulthood? Similar to Study 1, a binary logistic regression revealed that childhood fire exposure did not significantly predict the probability of participants listing a positive affective association with fire $(B=.05, p>.50)$. However, the 10 -item scale assessing positive affective reactions to fire allowed us to measure the relationship between childhood exposure and a continuous variable. The interrelationships between fire positivity and other variables of interest are summarized in Table 1. Contrary to the exposure hypothesis,

Table 1

Zero-Order Correlations Between Variables of Interest From Study 2

\begin{tabular}{llccccc}
\hline \multicolumn{1}{c}{ Variable } & 1 & 2 & 3 & 4 & 5 & 6 \\
\hline 1. Fire positivity & - & & & & & \\
2. Exposure before 10 & $.25^{* *}$ & - & & & & \\
3. Interest before 10 & $.52^{* *}$ & $.26^{* *}$ & - & & & \\
4. Entertainment between age 10-18? & $.47^{* *}$ & $.18^{* *}$ & $.52^{* *}$ & - & & \\
5. Exposure frequency past year & .11 & $.33^{* *}$ & .09 & .00 & - & .03 \\
6. Negative experience? & .07 & .09 & .12 & $.16^{*}$ & .03 & -11 \\
7. Ease of lighting fire & $.42^{* *}$ & $.35^{* *}$ & $.32^{* *}$ & $.29^{* *}$ & .12 & - \\
\hline
\end{tabular}

${ }^{*} p<.05 . \quad{ }^{* *} p<.01$. 
childhood fire exposure was positively associated with positive affective reactions, $r=.25$, $p<.001-$ a relationship that was inferentially identical for participants born in Alaska versus those who were not ( $r \mathrm{~s}=.27$ and .24 , respectively). This relationship was greater in magnitude for men, $r=.39, p=.01$ than for women, $r=.23, p<.001$; however, a follow-up multiple regression analysis revealed that the interaction between exposure and gender was nonsignificant $(p=.38)$. Also contrary to the hypothesis was a significant positive association between the affective fire scale and reported proficiency in starting a fire, $r=.42, p<$ .001 - a relationship similar in magnitude for men and women ( $r$ 's $=.51$ and .40 , respectively, $p \mathrm{~s}<.001)$.

Across the sample, $52 \%$ of participants reported having used fire for entertainment purposes (e.g., "fire play") at some point between the ages of 10 to 18 . Similar to previous research, a higher proportion of men $(63 \%)$ reported having engaged in this activity relative to women $(50 \%)$, although this difference was not significant $(p=.15)$. Relative to participants who did not report such behavior, participants who had used fire for entertainment also reported significantly higher interest in fire before age $10, t=9.28, p<.001$, and had significantly higher fire positivity scores, $t=8.17, p<.001$. Surprisingly, participants who reported using fire for entertainment reported more exposure to fire before the age of $10, t=2.71, p=.007$. This result suggests that additional exposure to fire in early childhood may not help curb the risk of adolescent fire play in populations such as that sampled here.

Interestingly, for both men and women, higher fire positivity scores were not associated with exposure to fire in the past year, nor was childhood interest associated with current fire exposure. These results suggest minimal selfselection for exposing oneself to fire. Exposure before age 10 was similarly related to reported interest before age 10, $r=.26, p<.001$, and with reported proficiency in starting a fire, $r=$ $.35, p<.001$.

To test the unique predictive effects of early exposure to fire on current fire positivity, we performed a multiple regression predicting fire scale scores from exposure before age 10, exposure in the past year, fire-starting proficiency, gender, and whether participants reported a neg- ative experience with fire in their lifetimes. Results from this regression revealed a significant predictive effect of exposure before age $10(B=$ $.13, p=.03)$, as well as a unique effect of reported proficiency $(B=.33, p<.001)$. Exposure to fire in the past year, gender, and negative experiences with fire did not emerge as significant predictors of fire positivity as assessed by the fire scale, $p \mathrm{~s}>.50$.

A final multiple regression investigated the potential unique predictors of actual fire behavior. We entered fire exposure before age 10, interest before age 10, fire-starting proficiency, gender, and whether participants reported a negative experience with fire in their lifetimes as predictors of frequency of exposure to fire within the past year. The unique predictive effects of exposure to fire before age 10 were significant, $B=.33, p=.001$. None of the other predictors were significant, $p \mathrm{~s}>.12$. These results further suggest that interest in fire, either early in life or in adulthood, does not lead individuals in this population to self-select higher levels of fire exposure.

\section{General Discussion}

Two tentative conclusions can be drawn from these results. First, in the populations sampled, people's affective associations with fire appear to be overwhelmingly positive. This was the case in both a predominantly urban Vancouver sample, and in an Anchorage sample comprised of a greater percentage of people from a rural background. Second, inconsistent with predictions from the exposure hypothesis, these positive affective reactions do not appear to be dampened by exposure to fire in childhood. In fact, results from Study 2 suggest that the reverse may be true-that a higher frequency of exposure to fire in childhood, as well as greater proficiency with fire use, is related to higher positivity toward fire in adulthood, and a higher likelihood of having used fire for entertainment purposes in adolescence. This relationship was independent of any potential effects of negative experiences with fire or recent frequency of fire exposure.

How are these results to be reconciled with findings reported in Fessler (2006), who found little or no psychological proclivity toward fire once proficiency in fire manipulation was attained? Several possibilities exist. 
First, Fessler (2006) based his conclusions on ethnographers' recollections, and these may simply have been inaccurate. The data used were retrospective accounts, and none of the ethnographers had set out to investigate psychological aspects of fire. If these accounts were indeed inaccurate, then conducting focused investigations such as that reported here in smallscale subsistence societies should reveal similar patterns across divergent cultures.

Second, it is possible that the levels of fire exposure reported in our samples were still below the threshold required by the learning mechanism postulated by the exposure hypothesis. The ethnographic accounts provided in Fessler (2006) were obtained from societies in which, barring unusual circumstances, people were exposed to fire throughout the course of daily activities. Conversely, although the current Anchorage sample (Study 2) reported much higher exposure to fire than our Vancouver sample (Study 1), still, only $8.5 \%$ of respondents reported even near-daily exposure to fire throughout childhood. Repeated exposure to a stimulus often produces increases in the reinforcing properties of that stimulus (sensitization) before the onset of habituation, reflected in the commonly observed pattern of early increases in operant response rates before eventual decreases. Sensitization followed by habituation occurs not only with food reinforcers, but also with activities including painting, listening to music, and walking (Murphy, McSweeney, Smith, \& McComas, 2003). It is therefore possible that the moderate fire exposure experienced by participants in the current studies produced sensitization, whereas Fessler's (2006) sample experienced habituation.

The current samples, then, may not have provided a sufficient test of the exposure hypothesis; it remains possible that samples with higher average exposure to fire throughout childhood will indeed demonstrate the negative relationship between fire exposure and interest predicted by the exposure hypothesis. This possibility can be addressed by using the instruments developed here with both high-exposure and moderate-exposure populations, thereby potentially elucidating the boundary conditions for this relationship.

Third, it is possible that cultural models of the meaning of fire have a profound impact on psychological associations with fire, such that the difference between the present results and those in Fessler (2006) reflect real differences between societies-differences due not to exposure to fire, but to the cultural frames of reference within which interactions with, and responses to, fire occur. Specific characteristics of fire are differentially beneficial across cultures. The participants in the studies reported here inhabit high latitudes and relatively cold climates, which places the heat provided by fire at a premium. In this sense, the cultural experience of fire may be fundamentally different from those of the cultures reported by Fessler, given that these groups inhabit warmer climates, and have less necessity for fires as a source of heat. Future research will benefit from more rigorously investigating the importance of, and interest in, fire in equatorial societies, and in societies where fire is a necessity in daily life more generally.

At present, it is unclear which of the above three possibilities is correct. What is clear, however, is that these questions provide ample opportunity for future research in a surprisingly overlooked topic that lies at the intersection of human evolution, psychology, and culture.

The two samples reported here are also constrained by being comprised of a disproportionate amount of female participants. Previous research suggests that male and female children may have different experiences with fire, with boys being more likely to use fire for entertainment than girls (e.g., Perrin-Wallqvist \& Norlander, 2003). Although between-gender difference were nonsignificant in the current studies, it is unclear whether this nonsignificance is due more to relatively low statistical power than due to actual similarities in fire experience between girls and boys.

Different cultural models of the meaning of fire also likely vary in their attribution of fire use being a prototypically male or female activity. These differential cultural models likely contribute to any gender difference that exists in fire interest, exposure, or reported proficiency. In many of the cultures reported by Fessler (2006), for example, women more commonly tend to fire-related activities than men. In the current samples, on the other hand, any between-gender differences tend toward men reporting more exposure to, and proficiency with, fire. Overall, however, the between-gender 
comparisons reported here offer limited inferential value to address this possibility.

The present results have potential implications for public safety interventions. Fessler (2006) suggested that opportunities to safely manipulate fire in childhood may curb adolescent firesetting tendencies. Our findings, however, suggest that such interventions may be ineffectual or even counterproductive. In our second, more exhaustive study, participants who reported using fire for entertainment purposes during adolescence reported significantly more exposure to fire before adolescence. Although this inference is limited by the possibility that this sample as a whole does not have a level of fire exposure necessary to satisfy the boundary conditions within which the exposure hypothesis operates, from the perspective of officials and educators responsible for enhancing public safety in technologically modern nation-states, such considerations are largely irrelevant-what matters is that, in these societies, enhancing fire exposure early in life is unlikely to curb subsequent dangerous fire-related behaviors, and may well exacerbate them.

Despite the damage and destruction that it causes, and despite the fact that fire has very limited nonindustrial utility today, fire remains a source of fascination in modern societies. This psychological proclivity demands comprehensive explanations, whether they concern universal features of human minds, parochial features of cultural values, or a combination of the two. To date, however, the current investigation represents one of the few inquiries into the psychology of fire. We hope that this state of affairs will change as more scholars begin to appreciate the importance of fire throughout human evolution.

\section{References}

Barrett, H. C. (2005). Adaptations to predators and prey. In D. M. Buss (Ed.), The evolutionary psychology handbook (pp. 200-223). Hoboken, NJ: Wiley.

Barrett, H. C., \& Broesch, J. (2012). Prepared social learning about dangerous animals in children. Evolution and Human Behavior, 33, 499-508. http:// dx.doi.org/10.1016/j.evolhumbehav.2012.01.003

Berna, F., Goldberg, P., Horwitz, L. K., Brink, J., Holt, S., Bamford, M., \& Chazan, M. (2012). Microstratigraphic evidence of in situ fire in the Acheulean strata of Wonderwerk Cave, Northern
Cape province, South Africa. Proceedings of the National Academy of Sciences, USA of the United States of America, 109, E1215-E1220. http://dx .doi.org/10.1073/pnas.1117620109

Brain, C. K. (1993). The occurrence of burnt bones at Swartkrans and their implications for the control of fire by early hominids. In C. K. Brain (Ed.), Swartkrans: A cave's chronicle of early man (pp. 243249). Pretoria, South Africa: Transvaal Museum.

Broesch, J., Barrett, H. C., \& Henrich, J. (2014). Adaptive content biases in learning about animals across the life course. Human Nature, 25, 181199. http://dx.doi.org/10.1007/s12110-014-9196-1

Brown, D. E. (1991). Human universals. New York, NY: McGraw-Hill.

Burton, F. D. (2009). Fire: The spark that ignited human evolution. Albuquerque, NM: University of New Mexico Press.

Ó Ciardha, C., \& Gannon, T. A. (2012). The implicit theories of firesetters: A preliminary conceptualization. Aggression and Violent Behavior, 17, 122-128. http://dx.doi.org/10.1016/j.avb.2011.12 .001

Cotterall, A. J. (2003). A survey of the disposition, affinity, interest and experience with fire of students in the city of Greater Sudbury (Unpublished M. A. thesis). Laurentian University, Sudbury, Ontario, Canada.

Fessler, D. M. T. (2006). A burning desire: Steps toward an evolutionary psychology of fire learning. Journal of Cognition and Culture, 6, 429451. http://dx.doi.org/10.1163/156853706 778554986

Garcia, J., Ervin, F. R., \& Koelling, R. A. (1966). Learning with prolonged delay of reinforcement. Psychonomic Science, 5, 121-122. http://dx.doi .org/10.3758/BF03328311

Goren-Inbar, N., Alperson, N., Kislev, M. E., Simchoni, O., Melamed, Y., Ben-Nun, A., \& Werker, E. (2004). Evidence of hominin control of fire at Gesher Benot Ya'aqov, Israel. Science, 304, 725727. http://dx.doi.org/10.1126/science. 1095443

Hall, J. R. J. (2003). Children playing with fire. Quincy, MA: National Fire Protection Association.

Kolko, D. J. (2002). Handbook on firesetting: Children and youth. San Diego, CA: Academic Press.

Murphy, E. S., McSweeney, F. K., Smith, R. G., \& McComas, J. J. (2003). Dynamic changes in reinforcer effectiveness: Theoretical, methodological, and practical implications for applied research. Journal of Applied Behavior Analysis, 36, 421438. http://dx.doi.org/10.1901/jaba.2003.36-421

Naeher, L. P., Brauer, M., Lipsett, M., Zelikoff, J. T., Simpson, C. D., Koenig, J. Q., \& Smith, K. R. (2007). Woodsmoke health effects: A review. Inhalation Toxicology, 19, 67-106. http://dx.doi.org/ $10.1080 / 08958370600985875$ 
Ohman, A., Fredrikson, M., Hugdahl, K., \& Rimmö, P. A. (1976). The premise of equipotentiality in human classical conditioning: Conditioned electrodermal responses to potentially phobic stimuli. Journal of Experimental Psychology: General, 105, 313-337. http://dx.doi.org/10.1037/00963445.105.4.313

Perrin-Wallqvist, R., Archer, T., \& Norlander, T. (2004). Adolescents' fire-setting awareness under boredom: Relation to personality variables. Psychological Reports, 94, 863-871. http://dx.doi .org/10.2466/pr0.94.3.863-871

Perrin-Wallqvist, R., \& Norlander, T. (2003). Firesetting and playing with fire during childhood and adolescence: Interview studies of 18-year-old male draftees and 18-19-year-old female pupils. Legal and Criminological Psychology, 8, 151-157. http://dx.doi.org/10.1348/135532503322362933

Pinsonneault, I. L. (2002). Fire safety education and skills training. In D. J. Kolko (Ed.), Handbook on firesetting in children and youth (pp. 219-260). San Diego, CA: Academic Press. http://dx.doi.org/ 10.1016/B978-012417761-1/50013-7

Platek, S. M., Gallup, G. G., Jr., \& Fryer, B. D. (2002). The fireside hypothesis: Was there differential selection to tolerate air pollution during human evolution? Medical Hypotheses, 58, 1-5. http://dx.doi.org/10.1054/mehy.2001.1385

Pyne, S. J., \& Goldammer, J. G. (1997). The culture of fire: An introduction to anthropogenic fire his- tory. In J. S. Clark, H. Cachier, J. G. Goldammer, $\&$ B. J. Stocks (Eds.), Sediment records of biomass burning and global change (pp. 71-114). Berlin, Germany: Springer-Verlag. http://dx.doi.org/ 10.1007/978-3-642-59171-6_5

Siegal, M., Fadda, R., \& Overton, P. G. (2011). Contamination sensitivity and the development of disease-avoidant behaviour. Philosophical Transactions of the Royal Society of London Series B, Biological Sciences, 366, 3427-3432. http://dx.doi .org/10.1098/rstb.2011.0036

Sillen, A., \& Hoering, T. (1993). Chemical characterization of burnt bones from Swartkrans. In C. K. Brain (Ed.), Swartkrans: A cave's chronicle of early man (pp. 243-249). Pretoria, South Africa: Transvaal Museum.

Simonsen, B., \& Bullis, M. (2001). Fire interest survey: Final report. Salem, OR: Oregon Office of the State Fire Marshal.

Wiessner, P. W. (2014). Embers of society: Firelight talk among the Ju/'hoansi Bushmen. Proceedings of the National Academy of Sciences, USA of the United States of America, 111, 14027-14035. http://dx.doi.org/10.1073/pnas.1404212111

Wrangham, R. (2009). Catching fire: How cooking made us human. New York, NY: Basic Books.

Received August 24, 2014

Revision received December 3, 2014 Accepted December 15, 2014 